\title{
Size and Isotope Effects of Helium Clusters and Droplets: Identification of Surface and Bulk-Volume Excitations
}

\author{
Klaus von Haeften, ${ }^{* \dagger}$ Tim Laarmann, ${ }^{\ddagger}$ Hubertus Wabnitz, ${ }^{\ddagger}$ Thomas Möller, ${ }^{\S}$ and Karin Fink ${ }^{\prime \prime}$ \\ ${ }^{\dagger}$ Department of Physics and Astronomy, University of Leicester, Leicester LE1 7RH, United Kingdom \\ ${ }^{\ddagger}$ Deutsches Elektronen-Synchrotron DESY, 22603 Hamburg, Germany \\ ${ }^{\S}$ Institut für Optik und Atomare Physik, Technische Universität Berlin, Hardenbergstrasse 36, 10623 Berlin, Germany \\ "Institut für Nanotechnologie, Karlsruher Institut für Technologie, Postfach 3640, 76021 Karlsruhe, Germany
}

\begin{abstract}
We report a comprehensive investigation of the electronically excited states of helium clusters and droplets of sizes ranging from a few to several $10^{7}$ atoms using time-resolved fluorescence excitation spectroscopy and quantum chemical ab initio calculations. We employ various approaches for our analysis considering the lifetime-dependence of the fluorescence intensity, spectral shifts, intensity scaling with cluster size, isotopic dependence, and density-dependence of the calculated electron wave function radii. A unique feature of helium clusters and droplets is their radially varying particle density. Our results show that short-lived fluorescence is sensitive to regions of increased density and probes excitations located in the bulk volume, whereas long-lived fluorescence is sensitive to regions of reduced density such as for small clusters or for the surface of large droplets. Spectra of ${ }^{3} \mathrm{He}$ droplets serve as a reference for low density, but are free from contributions of small clusters. This allows us to distinguish regions of reduced density as these can be due to both surface states or small clusters. Our analysis reveals a picture where spectral features are related to regions of different density due to isotopic composition, cluster size, and surface or bulk volume location of the excitations. The $2 s$ and $2 p$ related excitations appear as blueshifted wings for small clusters or for excited atoms within the surface layer, whereas in the bulk-volume of large droplets, they appear as distinct bands with large intensities, dominating the entire spectrum. Excitations at energies higher than $23 \mathrm{eV}$ are unambiguously assigned to regions of low and medium density location within the deeper parts of the surface layer but show no relation to the bulk volume. Our findings support the idea that in liquid helium high-lying states and, in particular, Rydberg states are quenched in favor of the $2 s$ and $2 p$ excitations.
\end{abstract}

\section{INTRODUCTION}

The helium atom is the simplest example of a many-electron atom. In light of the simplicity and model character of helium atoms one would naturally be interested to learn how the electronically excited states become modified when an excited helium atom is placed near one, two, or more helium atoms. A straightforward experiment to investigate this problem is to produce a beam of helium clusters of variable size and to photoexcite these clusters in the vacuum ultraviolet (VUV) spectral range using synchrotron radiation. Such an experiment probes a disordered arrangement of atoms because helium clusters and droplets in a free beam are always liquid. A molecular beam of clusters is optically thin and absorbs very little light, but helium clusters have a large fluorescence yield. Therefore, photofluorescence yield detection is the method of choice. The first experiments of ${ }^{4} \mathrm{He}$ clusters of variable size using a VUV fluorescence light detector identified a number of bands and it was found that the energies of these excitations, unlike the electronic excitations of heavy rare gas clusters, ${ }^{1,2}$ did not fit to a Wannier-type excitonic series. ${ }^{3}$ Subsequent experiments investigated fluorescence decay channels in different wavelength regions, ${ }^{4,5}$ the effect of helium particle density, ${ }^{6}$ fluorescence in cavities within large helium droplets, ${ }^{7}$ and the possible Rydberg nature of the excited states of small helium clusters. ${ }^{8}$ First quantum chemical calculations of an octahedral model cluster reported the energies of the $n=2,3$, and 4 states of the central atom as a function of the internuclear separation in the octahedron. ${ }^{9}$ Electronic spectra simulations of a perturbed octahedron as well as an $N=25$ atom-large cluster ${ }^{10}$ were able to reproduce previously reported experimental data of small clusters. ${ }^{6}$

Despite these efforts, we still have an incomplete picture of the electronically excited states of large helium clusters. The number of states increases dramatically with size making the computation and interpretation of the results laborious. The availability of experimental data that cover the entire size range of helium clusters and droplets is prerequisite as a benchmark for testing theoretical interpretation and likewise prerequisite in providing evidence for empirical interpretation. Furthermore, the comprehensive data set presented in this paper shows that each helium cluster size has a specific spectral fingerprint. A unique feature of helium clusters and droplets is the 6-7 $\AA$ thick surface region where the density drops smoothly from the bulk value to zero. ${ }^{11,11,12}$ With regard to electronically excited states, such a surface layer represents a

Special Issue: J. Peter Toennies Festschrift

Received: January 26, 2011

Revised: $\quad$ March 16, 2011

Published: April 05, 2011 
region of gradually increasing perturbation where the character of excited atoms is expected to change from gas-type to condensed matter behavior. This crossover should produce a specific signature in the absorption and excitation spectra, which our paper aims to investigate. We report VUV fluorescent yield photoexcitation spectra of ${ }^{3} \mathrm{He}$ and ${ }^{4} \mathrm{He}$ clusters and large droplets covering the size range from $N=1$ to $N>$ $10^{7}$ for ${ }^{4} \mathrm{He}$ clusters and $\mathrm{N}>10^{4}$ for ${ }^{3} \mathrm{He}$ droplets. Compared to the early days of such research on helium clusters, reliable data of the average cluster sizes produced at specific source conditions is now readily available. ${ }^{11,13}$ The availability of this data allows a much better assignment of sizes than was possible in the early studies.

Our method is to investigate both stable isotopes of helium, ${ }^{3} \mathrm{He}$ and ${ }^{4} \mathrm{He}$. Clusters of the two isotopes differ in their particle density, liquid ${ }^{3} \mathrm{He}$ being about $75 \%$ the density of ${ }^{4} \mathrm{He}$. A second significant difference is that beams of ${ }^{3} \mathrm{He}$ clusters are free of small clusters, because for these, no bound states exist, resulting in growth mechanisms entirely different from ${ }^{4} \mathrm{He}$. Most or all atoms in a small helium cluster belong to the surface region and exhibit a reduced particle density with respect to the bulk. The similarities between small helium clusters and the surface region of large helium clusters make it difficult to distinguish between the two, so ${ }^{3} \mathrm{He}$ cluster beams are therefore very helpful in the assignment of spectral features. Features that reflect a reduced density region can be assigned to the clusters' surfaces, while small clusters for which ${ }^{4} \mathrm{He}$ clusters and droplets always accompany the beam can be excluded. In addition to looking at different isotopes, we also employ time-resolved fluorescence yield detection to record excitation spectra related to specific relaxation channels. ${ }^{14-16}$ We observe that short-lived fluorescence excitation spectra show features predominantly related to the bulk, while the opposite holds for the long-lived fluorescence. This difference allows for unambiguous assignment of surface features. As our work for the first time comprehensively explores the effect of size, it also represents an important benchmark for theory as well as for other experiments with helium clusters in this energy range. ${ }^{17-21}$

\section{口 EXPERIMENTAL SECTION}

The experiments were performed at the synchrotron light source DORIS III at HASYLAB/DESY using the experimental end-station CLULU at beamline I. The beamline is equipped with a $15^{\circ}$ normal incidence McPherson monochromator for VUV light and differential pumping stages providing windowless illumination of the free helium cluster beam. A platinum/ osmium-coated grating was used for excitation of helium clusters. The photon flux for this combination was measured earlier. ${ }^{14}$ In the relevant range from 19 to $26 \mathrm{eV}$ the spectral intensity was to a good approximation constant. Therefore, after conversion of the data into $\mathrm{eV}$, no intensity normalization was performed. The spectra presented in this paper are the raw data converted into $\mathrm{eV}$. Spectra were recorded using $100 \mu \mathrm{m}$ wide entrance and exit slits giving a resolution of $26 \mathrm{meV}$.

The VUV fluorescence yield was recorded by photon counting using an XP 2020 photomultiplier (Valvo/Philips) in air. The VUV photons were converted to the spectral sensitivity range of the photomultiplier using a plate vacuum coated with a thin film of sodium salicylate, just before a window and the photomultiplier. This technique is simple and it was found that the set up was insensitive to visible photons emitted from the helium clusters. ${ }^{8}$ Absorption of a photon leads to a good approximation to fluorescence of precisely one VUV photon whose registration can be taken as a measure for photo absorption. ${ }^{14}$ Limitations exist for high excitation energies where autoionization is possible and also for very large helium droplets where more than one excitation per synchrotron light pulse may occur. In this case, annihilation of the type $\mathrm{He}^{*}+\mathrm{He}^{*} \rightarrow \mathrm{He}+\mathrm{He}^{+}+e$ is possible. ${ }^{22}$ Time-resolved excitation spectra were recorded by counting photons selectively in "time-windows" after the excitation pulse of the synchrotron radiation using Time-To-Amplitude Converters (TAC) and Constant-Fraction-Discrimination for gating the signals. A time window of 3-18 ns after the excitation pulse was set to measure the excitation of short-lived fluorescence, and a time-window of $40-172$ ns was set to record long-lived fluorescence. The time resolution was limited by the use of sodium salicylate as wavelength converter and estimated to be at least $\sim 5 \mathrm{~ns}$.

The CLULU end station has been described in detail ${ }^{14}$ and modifications made for fluorescent spectroscopic experiments of helium clusters have been reported in several publications. ${ }^{3,5,8}$ Therefore, here we will only explain the most important features relevant to this paper. ${ }^{3} \mathrm{He}$ and ${ }^{4} \mathrm{He}$ clusters and droplets were produced using supersonic expansion of helium gas through an orifice of $5 \mu$ in diameter (Frey GmbH, Berlin) into the vacuum. When the expensive isotope ${ }^{3} \mathrm{He}$ was used, the gas was recycled, that is, collected from the pump exhausts, compressed, and purified. Details are described in the literature. ${ }^{8}$ The CLULU end station consists of a helium cluster source cooled by a continuous flow cryostat (Cryovac) and fluorescence detectors with different characteristics. The cluster source allows the nozzles to be cooled down to $6 \mathrm{~K}$ and for stagnation pressures up to 50 bar to be applied. Nozzles with different diameters can be attached to the cold head. The temperature is controlled by resistive heating using a Lake Shore cryo controller, a DT-470 cryogenic diode, and an ohmic resistive heater. The absolute tolerance of the DT-470 diode was $\pm 0.5 \mathrm{~K}$, according to manufacturer specifications. As the cluster size is a central issue in this paper, and as the nozzle temperature $T_{0}$ is critical for the size, $T_{0}$ is indicated for every spectrum.

The helium cluster beam was directly excited with monochromatic synchrotron radiation without using a skimmer to maximize the cluster density. The focus of the beamline was about 5 $\mathrm{mm}$ wide and extended from $0.5 \mathrm{~mm}$ after the nozzle to $5.5 \mathrm{~mm}$ downstream. The cluster source was mounted on an $\mathrm{x}, \mathrm{y}, \mathrm{z}$ manipulator to maximize overlap of the cluster beam with the synchrotron light but to minimize stray-light from light scattering off the nozzle. Fluctuations of the positron beam position in the storage ring required adjustment of the nozzle position at each start of an experimental run. The optimum distance was adjusted after evaluation of seven spectra, each recorded at different nozzle-focus separation.

The entire vacuum chamber was pumped using a $2200 \mathrm{~L} / \mathrm{s}$ turbo pump (Pfeiffer). The turbo pump was backed by a roots/ rotary stack of $250 \mathrm{~L} / \mathrm{s}$ and $40 \mathrm{~m}^{3} / \mathrm{h}$ pumping speed, respectively. The pressure did not exceed $2 \times 10^{-3}$ mbar (corrected for helium ionization efficiency).

Quantum Chemical Calculations. Quantum chemical calculations using the Bochum suite of wave function based quantum chemical ab initio programs ${ }^{23-25}$ were performed for an octahedral helium model cluster in which the central atom was excited and the bond lengths between the center and the "surface" atoms were simultaneously varied. This bond length variation aimed to 


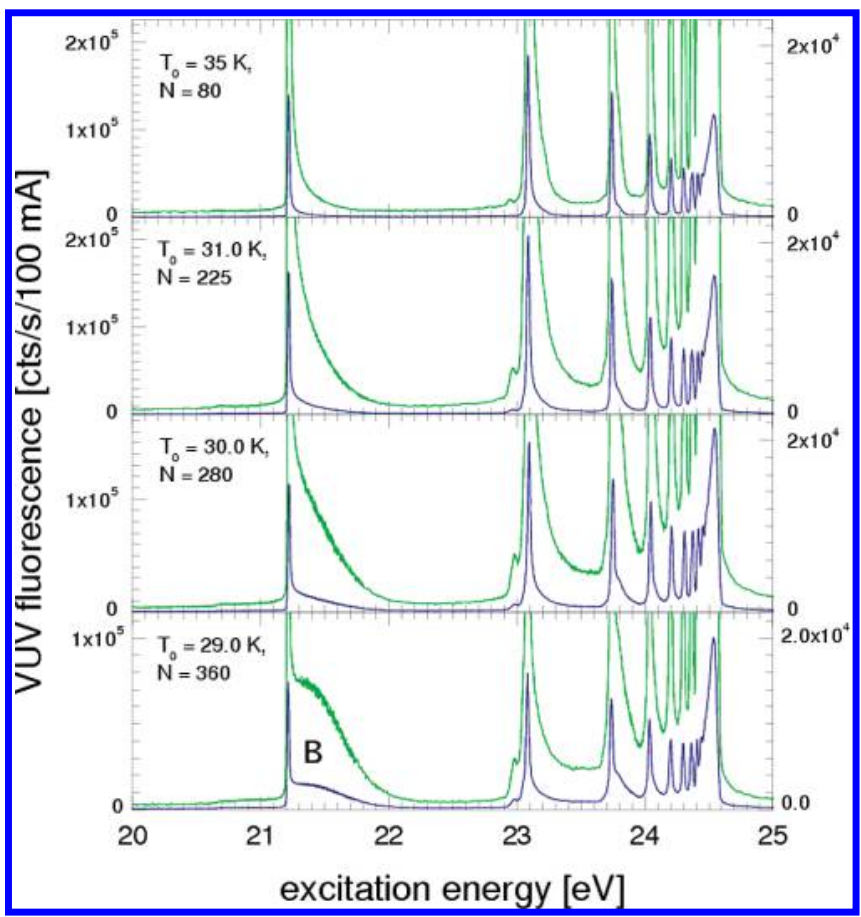

Figure 1. Fluorescence excitation spectra of ${ }^{4} \mathrm{He}$ clusters of $80-360$ atoms on average (blue line). The fluorescence yield was measured with a VUV-sensitive detector and may be taken as a measure for the photoabsorption (see text). The spectra are shown using two scales to visualize finer details (green line, belongs to right scale) of the spectral features. The intense sharp lines are due to $2 p, 3 p, 4 p \leftarrow 1 s$ transitions.

simulate the effect of varying density. Excitation energies were calculated using the self-consistent field (SCF) method. The orbitals were localized at the central atom using a Foster-Boys localization. Valence state excitations were calculated using the state averaged complete active space SCF method (CASSCF) and dynamic correlations were taken into account using the multiconfiguration coupled electron pair approximation (MCCEPA) to the multireference coupled cluster method. ${ }^{25}$ Rydberg states with $n \geq 3$ were calculated using the frozen-core approximation. ${ }^{26,27} \mathrm{We}$ used the $10 \mathrm{~s}$ basis set of Huzinaga ${ }^{28}$ contracted to $6 \mathrm{~s}$. This basis set was extended by $\mathrm{p}(2.0,0.7,0.2)$ and $\mathrm{d}(1.4,0.4)$ polarization functions as well as by semi-diffuse $\mathrm{s}(0.03)$ and $\mathrm{p}(0.07)$ functions. For the $\mathrm{He}$ atom at which the core excitation took place it was necessary to use the uncontracted 10s basis set to allow for changes in the orbital shape of the 1s orbital. Furthermore, a Rydberg basis of Kaufman et al. ${ }^{29}$ which was able to describe Rydberg states up to $n=5$ was added at this atom. Details are described in ref 9. The frozen-core calculations allowed us to deduce the average electronic wave function radius $\langle r\rangle$.

\section{RESULTS AND DISCUSSION}

All spectra presented in this paper were recorded using a $5 \mu \mathrm{m}$ orifice and a stagnation pressure of 40 bar. The average cluster/ droplet size was varied by changing the source temperature only. For calibration of the monochromator the wavelength was repeatedly scanned past the $3 p^{1} P \rightarrow 1 s^{1} S$ atomic line at $537 \AA$ at stagnation temperatures $T_{0}$ between 81 and $96 \mathrm{~K}$. At these temperatures, only sharp lines were observed, showing that the features were purely due to atoms. The average cluster sizes $N$ in the region from 35 to $22 \mathrm{~K}$ were determined using an exponential-decay fit to the data reported by Harms et al. ${ }^{13}$ together with our data point at $81 \mathrm{~K}$ representing $N=1$ (see Table 2$)$. This turned out to be a robust approach, as shifting the $N=1$ data point to temperatures lower than $81 \mathrm{~K}$ did not change the sizes in the range between 35 and $22 \mathrm{~K}$ significantly. The fit to Harms' data was very good for $T_{0}<$ $20 \mathrm{~K}$, but produced larger sizes for higher $T_{0}$ at 22 and $24 \mathrm{~K}$. We suspect that the cross jet/pick-up method used by Harms et al. is less reliable for small helium droplet sizes where doping by the pick-up method becomes difficult. Using Hagena's formula for size determination ${ }^{30}$ was not an option because of significant deviations from the ideal gas approximation for helium at low temperatures. Spectra were then recorded at progressively lower temperatures, starting with $35 \mathrm{~K}$ (Figure 1).

Spectra of Small ${ }^{4} \mathrm{He}$ Clusters. The spectrum at $35 \mathrm{~K}$ shows intense sharp lines at the position on the $n p \leftarrow 1 s$ transitions accompanied by blue-shifted wings. The lines converge toward the atomic ionization limit at $24.56 \mathrm{eV}$ where they overlap strongly, giving rise to large intensities. The spectrum resembles that of dense helium gas in a cell ${ }^{31}$ with the difference that the blue-shifted wing between 21.2 and $22 \mathrm{eV}$ is continuous and does not end abruptly. The abrupt end observed for dense gas was interpreted as an increased density of states, indicating a flat-top region in the $\mathrm{He}-\mathrm{He}^{*}$ potential energy curves. The continuously progressing shift observed for small helium clusters shows that mixing with states higher than $n=2$ is very likely involved. Between 23.2 and $23.25 \mathrm{eV}$, the wing next to the $3 p$ line shows less continuity than the $2 p$ wing; a similar "bump" is observed for the wing of the $4 p$ line, indicating a region of increased density of states. Also, a small peak of the dipole-forbidden $3 s^{1} S \leftarrow 1 s^{1} S$ excitation at $22.92 \mathrm{eV}$ is seen as well as a shoulder at the position of the $4 s^{1} S \leftarrow 1 s^{1} S$ excitation at $23.67 \mathrm{eV}$. These transitions become allowed as the spherical symmetry of the electronic wave function is more and more broken as the atoms condense into molecules and clusters. Spectra at stagnation temperatures higher than $35 \mathrm{~K}$ were difficult to record as the nozzle temperature is more difficult to stabilize in this region. They do not show any substantial variation with regard to the spectrum at $35 \mathrm{~K}$. At $T_{0}=31 \mathrm{~K}$, corresponding to $N=225$, the blue-shifted wings further increase in intensity compared to the sharp lines filling the gap between the $3 p$ and $4 p$ features. The dipole forbidden $2 s^{1} S \leftarrow 1 s^{1} S$ excitation at $20.62 \mathrm{eV}$ can be identified as a step in the spectrum and a band expanding up to the $2 p$ line. The spectra evolve in a similar way when $T_{0}$ is further lowered. At $T_{0}=28 \mathrm{~K}$, a distinct feature, "band B", superimposed on the $2 p$ wing starts to emerge.

The overall intensity of the features decreases with increasing principal quantum number $n$. This is expected for Rydberg-type systems because the radial overlap between the ground state and excited state wave function decreases. For larger clusters, this decrease becomes less pronounced. A possible reason is mixing of states with stronger contributions from low-lying states. It indicates that Rydberg states inside large helium clusters are unlikely, as was suspected for $\mathrm{NO}$ in helium droplets. ${ }^{32}$ An exception is the intensity of the $2 p$ line. One would expect the atomic $2 p$ line to be strongest, but the geometry of our windowless differential pumping setup (which allows helium atoms to flow into the beamline) blocks excitation light at the atomic resonances. The resulting intensity available at the focus is very difficult to estimate, but this effect may qualitatively explain why the $2 p$ line, which has the greatest oscillator strength, is weaker than the $3 p$ line, especially for $N=80$ large clusters.

Ab Initio Calculations. Figure 2 shows calculated average radii of electronic wave functions of octahedral helium clusters as a function of the bond length using the frozen core approximation. 


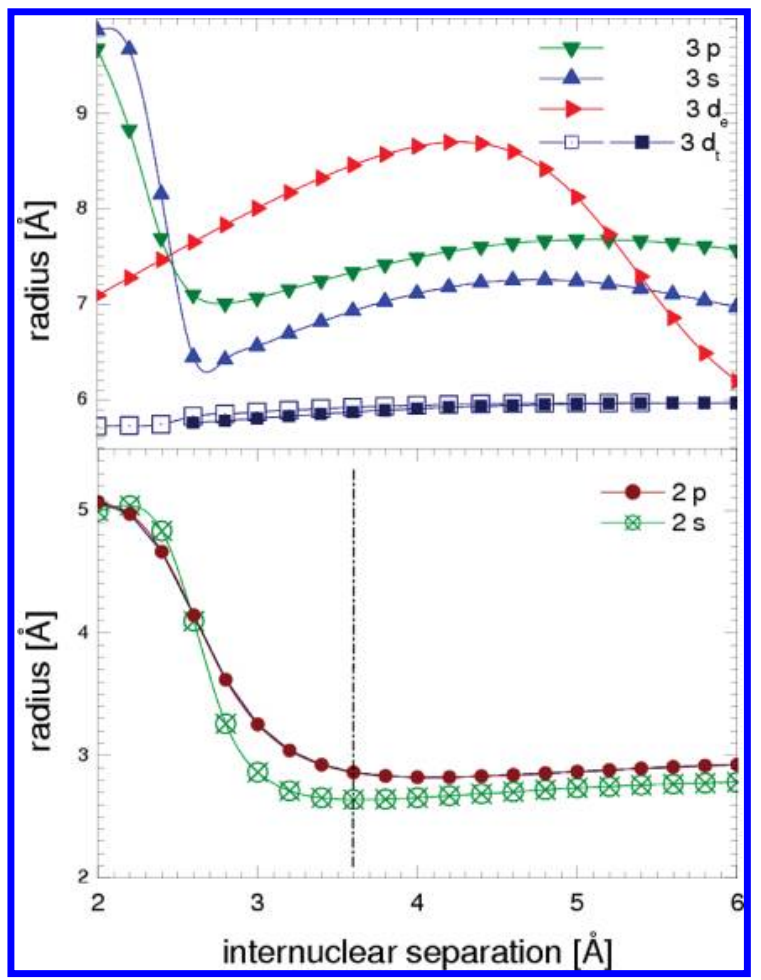

Figure 2. Calculated average radii of electronic wave functions using the frozen-core approximation as a function of the internuclear separation $R$ of the helium atoms in an octahedral model helium cluster. The dashdotted line shows the average internuclear separation in bulk-liquid ${ }^{4} \mathrm{He}$ and in the center of large helium droplets. All internuclear separations larger than this minimum value occur in helium clusters and droplets (see text). The top figure shows wave function radii associated with $n=3$ and the bottom figure shows radii associated with $n=2$. The wave functions are labeled in the atomic limit as $2 s, 2 p, 3 d, 3 s$, and $3 p$. The $3 d$ states split into $t_{2 g}$ and $e_{g}$ states due to the octahedral field. For the $n=2$ levels accurate energies were calculated using the CASSCF method and compared with the frozen core result (see text). For the $n=3$ levels only the frozen-core approximation was used. For small internuclear separations $(R<3 \AA)$ the wave functions develop strong Rydberg character with large electron radii. For $n=3$, some levels were found to have valence character throughout the entire bond-length range.

The internuclear separation in helium clusters is not constant and varies due to the decaying number density at the surface. The dashdotted line shows the average internuclear separation in bulk-liquid ${ }^{4} \mathrm{He}$ or in the center of large helium droplets which represents the lower limit. The wave function radii that belong to internuclear separations on the right-hand side of the dash-dotted line Figure 2 are those that can be expected in real helium clusters and droplets.

To test the validity of the frozen core approximation for lower energies we have calculated the energies of the $n=2$ states using the more accurate CASSCF method. We found very good qualitative agreement between CASSCF and frozen core, the only difference being a constant offset in energy, and conclude that the average wave function radii $\langle r\rangle$ produced by the frozen core method provide a convenient, and qualitatively correct description. The wave functions are labeled in the atomic limit as $2 s, 2 p, 3 d, 3 s$, and $3 p$. The $3 d$ states split into $t_{2 g}$ and $\mathrm{e}_{g}$ states due to the octahedral symmetry of the ligand field. This splitting is an artifact due to our choice of the model cluster geometry. For the $2 s$ and $2 p$ states two regions can be identified. Internuclear separations smaller than $3 \AA$ produce large radii increasing up to

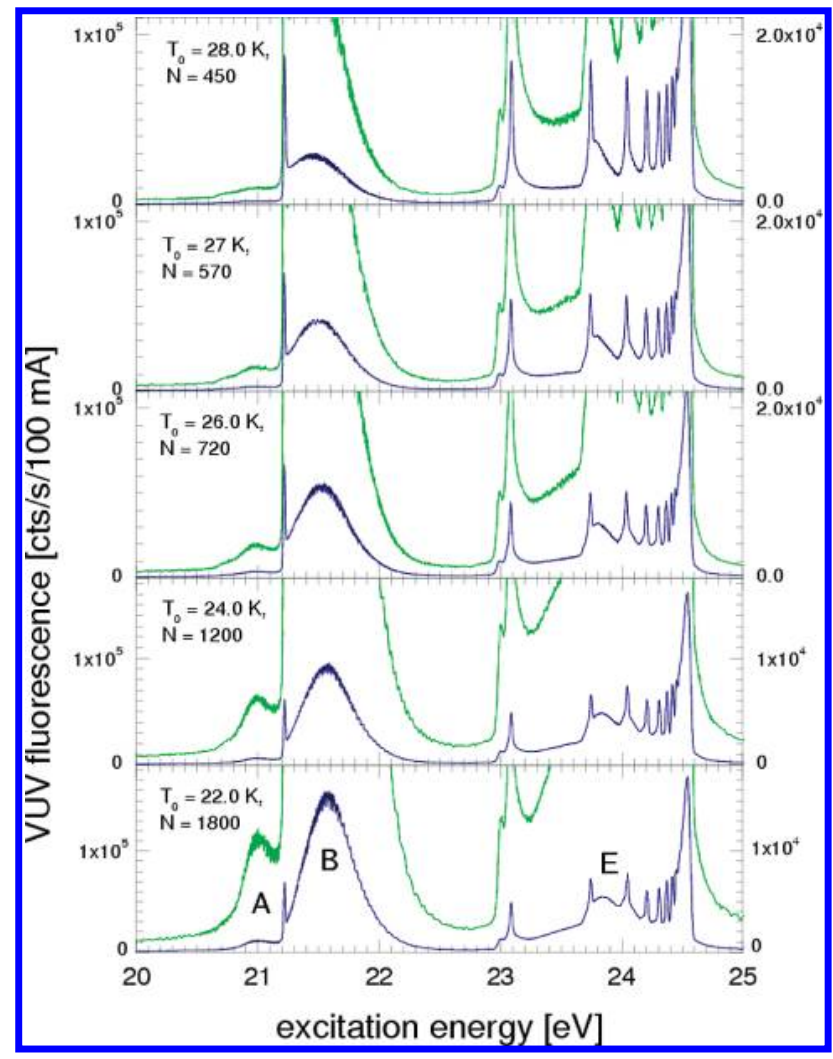

Figure 3. Fluorescence excitation spectra of ${ }^{4} \mathrm{He}$ droplets containing 450 to 1800 atoms on average. This size regime is characterized by the appearance of band A at $20.95 \mathrm{eV}$ and band B at $21.57 \mathrm{eV}$ (see text). Another band with a distinct maximum can be identified at $23.8 \mathrm{eV}$ (band E, see text and Table 1).

$5 \AA$ A Such small internuclear separations are only of importance if during excitations the nuclei are very close, which incidentally happens because of the large zero point motion amplitude. Average internuclear separations smaller than $3.56 \AA$ do not exist. The crossover at $3 \AA$ shows qualitatively that the electronic wave function can easily expand and develop Rydberg character if the internuclear separation is smaller than the radius of the electronic wave function. This is a realistic scenario for small helium clusters where most of the atoms are at the surface, and for excited states where the principal quantum number is larger than 3, because here Rydberg-type excited wave functions exist that are greater than the internuclear separation. These wave functions can expand into free space or, if $n$ is large enough, over the entire cluster. The same holds for atoms at the surface of large droplets, particularly for high $n$. Here the excited electron density and the atomic density are low and perturbation is minimized.

Clusters with bond lengths greater than $3 \AA$ produce radii of $2.8 \AA$ almost constant in size over the entire bond length range, showing that the character of the electronic wave function changes from Rydberg-type to valence-type. The decrease in radii observed when the internuclear separation is decreased from 6 to $3.5 \AA$ shows a slight compression of the wave function; a consequence of increasing perturbation by the electrons of the $1 s$ orbitals of the neighboring ground state atoms. For $n=3$ states a similar trend is observed: for two states the electron wave function radii increase rapidly when internuclear separation becomes smaller than $2.7 \AA$ and is compressed in the intermediate region up to $5 \AA$. The $3 d_{\mathrm{t} 2 \mathrm{~g}}$ states have valence 
Table 1. Band Energies ${ }^{a}$

\begin{tabular}{ccccc}
$\begin{array}{c}\text { size } \\
\text { (atoms) }\end{array}$ & band B & $\begin{array}{c}\text { band B } \\
\text { (short-lived) }\end{array}$ & band E & $\begin{array}{c}\text { band E } \\
\text { (short-lived) }\end{array}$ \\
360 & & 21.39 & & \\
450 & 21.45 & 21.48 & 23.80 & \\
570 & 21.50 & 21.50 & 23.80 & 23.79 \\
720 & 21.52 & 21.53 & 23.80 & 23.80 \\
1200 & 21.58 & 21.57 & 23.82 & 23.83 \\
1800 & 21.58 & 21.57 & 23.84 & 23.86 \\
2900 & 21.57 & 21.57 & 23.85 & 23.88 \\
4650 & 21.55 & 21.57 & 23.86 & 23.90 \\
9200 & 21.52 & 21.52 & 23.86 & 23.91 \\
${ }^{a}$ In $[\mathrm{eV}]$. & & & & \\
\hline
\end{tabular}

character throughout the entire range of internuclear separations.

Summarizing, the calculations show two effects that are relevant for the interpretation of the excited states of small helium clusters and excited states in the surface region of large droplets: the $n=2$ wave functions are always smaller than the internuclear separations present in real helium clusters and droplets. The $n=3$ Rydberg wave functions have similar or larger radii than the internuclear separations depending on whether the excitation is located in a very small cluster, near the surface or in the bulk volume. The calculated energies of the octahedral model cluster $^{9}$ show that the perturbation of the $n=3$ states is small when the electron density of the perturbing ground state helium atoms is small. As a consequence, Rydberg states can develop. The same holds for states with $n$ larger than 3. All spectra shown in Figure 1 belong to a cluster size regime where in the bulk volume the density is not high enough to produce shifted bands (bands A and B) and where the surface is so dilute that Rydberg states with high principal quantum numbers can establish.

Midsized ${ }^{4} \mathrm{He}$ Clusters and Large ${ }^{4} \mathrm{He}$ Droplets. Figure 3 shows spectra of ${ }^{4} \mathrm{He}$ droplets containing $450-1800$ atoms. The spectral features in this size range are characterized by the appearance of discrete bands, notably at 20.95, 21.60, and $23.85 \mathrm{eV}$ and also, though much weaker, at $20.75 \mathrm{eV}$. The bands at 20.95 and $21.60 \mathrm{eV}$, named $\mathrm{A}$ and $\mathrm{B}$, respectively, ${ }^{3}$ as well as the band at $23.86 \mathrm{eV}$ (band E) ${ }^{3}$ shift very slightly toward higher wavelengths with increasing cluster size. Closer inspection of band A reveals a double-peak structure previously not reported. ${ }^{3}$ The peak at $20.75 \mathrm{eV}$ appears slightly blue-shifted from the dipole forbidden $2 s^{1} S \leftarrow 1 s^{1} S$ excitation at $20.62 \mathrm{eV}$ but does not shift further when the cluster size increases. Its intensity increases less strongly than the peak at $20.95 \mathrm{eV}$. The maximum of band B shifts from $21.45 \mathrm{eV}$ for $N=450$ to $21.58 \mathrm{eV}$ for $N=1200$; it then stays constant up to $N=2900$ and decreases again to $21.53 \mathrm{eV}$ for $N=9200$. The change of the maximum position between $N=$ 450 and 1200 reflects the size distribution of clusters in the beam. Roughly, for an average size $N$, the standard deviation $\Delta N$ (fwhm) is $N .^{13}$ We have superimposed the two spectra of $N=$ 280 and $N=1200$ and find the maximum at $20.51 \mathrm{eV}$. This value matches the energy of band $\mathrm{B}$ of $21.52 \mathrm{eV}$ found for $N=720$. The good match is consistent with a mean size of the clusters in the beam of $N=720$ containing $N=280$ and $N=1200$ clusters at equal proportions, which supports our size assignment.

Band $\mathrm{E}$ appears at a cluster size of $N=450$ at $23.80 \mathrm{eV}$ and starts shifting toward $23.86 \mathrm{eV}$ at $N=1200$. The maxima of bands $\mathrm{B}$ and $\mathrm{E}$ are listed in Table 1.

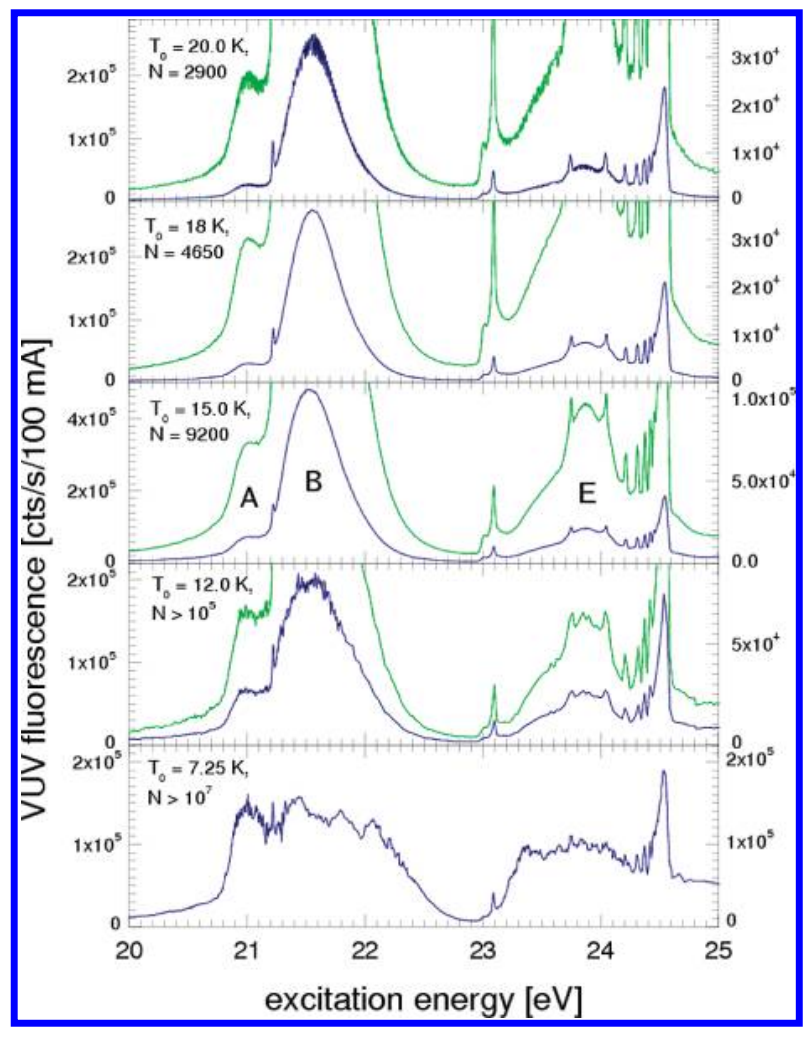

Figure 4. Fluorescence excitation spectra of ${ }^{4} \mathrm{He}$ droplets with sizes of $N=2900$ to $>10^{7}$. The size range from $N=2900$ to 9200 is characterized by the predominant growth of band $\mathrm{B}$ in intensity. The spectrum at $T_{0}=$ $20 \mathrm{~K}$ shows rapid modulations of the intensity of band $\mathrm{B}$, which are due to temperature fluctuations of about $0.5 \mathrm{~K}$ of the nozzle. At stagnation temperature below $15 \mathrm{~K}$ large helium droplets are formed by fragmentation from the liquid phase. ${ }^{33}$.

Figure 4 shows helium droplets in the size range from 2900 atoms to more than $10^{7}$. The size range from $N=2900$ to 9200 is characterized by a large intensity increase of band $\mathrm{A}$ and $\mathrm{B}$ compared to the rest of the spectral features. At $T=15 \mathrm{~K}$, corresponding to clusters of $N=9200$ atoms, one observes some flattening of the maximum of band $\mathrm{B}$, indicating saturation. This distortion continues when the source temperature is further lowered. At $T=12 \mathrm{~K}$-band $\mathrm{B}$ appears rugged and reduced in intensity compared to $T=15 \mathrm{~K}$. Supersonic expansions of helium at a stagnation temperature of $12 \mathrm{~K}$ proceed along isentropes that pass through the critical point. ${ }^{34}$ Droplets produced under these conditions are formed by fragmentation from the liquid phase rather than by condensation induced by three-body collision. ${ }^{34}$ The droplets therefore reach sizes that are distinctively larger. ${ }^{34,35}$ At $7.3 \mathrm{~K}$, the lowest temperature our apparatus was able to produce at $p_{0}=40 \mathrm{bar}$, the fluorescence yield spectrum appears heavily rugged, indicating strong nonlinear effects. We note that the intensity of the sharp $3 p$ resonance line at $23.10 \mathrm{eV}$ does not alter much when the stagnation temperature is lowered to $7.3 \mathrm{~K}$. From 20 to $7.3 \mathrm{~K}$ the intensity is almost constant, whereas from $T=35$ to $20 \mathrm{~K}$ the intensity decreases by about a factor of 4. At source conditions for $T_{0}<13 \mathrm{~K}$ two effects have to be considered: first, the ${ }^{4} \mathrm{He}$ droplets produced are very large. Depending on the wavelength droplets containing more than $10^{4}$ atoms are no longer optically thin, that is, the light transmitted through a droplet decays exponentially (due to Beer's law) because the droplets can have more than one excitation per synchrotron light 
Table 2. Relation between Stagnation Pressure $T_{0}$ and Droplet Size $N$ of ${ }^{4} \mathrm{He}$ Clusters and Droplets for Orifice Size $d_{0}=5$ $\mu \mathrm{m}$ and Stagnation Pressure $p_{0}=\mathbf{4 0} \mathrm{bar}$

\begin{tabular}{ccc}
$T_{0}$ & $N\left(\right.$ Harms et al. $\left.{ }^{13}\right)$ & fit (this work) \\
81 & & 1 \\
35 & & 80 \\
31 & & 225 \\
30 & & 280 \\
29 & & 360 \\
28 & & 450 \\
27 & & 570 \\
26 & 703 & 720 \\
24 & 1700 & 1200 \\
22 & 2617 & 1800 \\
20 & 4700 & 2900 \\
18 & 6130 & 4650 \\
17 & 8900 & \\
15 & 13000 & 9200 \\
13.5 & & $>10^{5}$ \\
12 & & $>10^{7}$ \\
7 & & \\
\hline
\end{tabular}

pulse. Second, the total flux of helium through the nozzle is so large that the beam is no longer optically thin. Furthermore, the large size gives rise for a much increased Mie scattering cross-section and this cross-section is further increased in the proximity of energy resonances. The reduced intensity observed for $T=7.3 \mathrm{~K}$ is therefore a consequence of both strong scattering and strong absorption at the boundary of the cluster beam. Both effects prevent the full light intensity from entering the focus.

${ }^{3} \mathrm{He}$ Droplets. ${ }^{3} \mathrm{He}$ droplets were produced at a stagnation pressure of $7 \mathrm{bar}$ and using the same $5 \mu \mathrm{m}$ orifice as for ${ }^{4} \mathrm{He}$ droplets. The excitation spectra of ${ }^{3} \mathrm{He}$ droplets are shown in Figures 5 and 6. Bound states of small ${ }^{3} \mathrm{He}$ clusters do not exist and therefore clusters grow through fragmentation from the liquid phase rather than via condensation seeds produced in three-body collisions. Clusters appear abruptly when the source is cooled below a threshold temperature. Figure 5 shows, for a source temperature of 11 $\mathrm{K}$, only sharp atomic resonance lines, for $10.6 \mathrm{~K}$ strongly rugged bands, and for $10.2 \mathrm{~K}$ fully developed bands. The shape and the intensity of the spectral features remain constant when the source temperature is further lowered to $9.0 \mathrm{~K}$. Further lowering of the temperature causes the features to distort and to lose intensity, similar to large ${ }^{4} \mathrm{He}$ droplets that were produced by fragmentation from the liquid phase. Measurements of the droplet size by Harms et al. using the cross beam deflection method reveal sizes that decrease slightly with decreasing source temperature. ${ }^{11}$ If we take the large distortion and intensity loss as indicators of the presence of very large droplets, our spectra show rather the opposite. We have therefore indicated the size only for source temperatures close to the onset of clustering where we believe the assignment by Harms et al. is reliable.

The spectral features of ${ }^{3} \mathrm{He}$ droplets show many similarities to ${ }^{4} \mathrm{He}$ : both bands A and B are observed, but with a different shift and width, as reported before. ${ }^{6}$ At higher energies differences to ${ }^{4} \mathrm{He}$ become larger, the $3 s$ feature is weaker in intensity compared to the $3 p$ atomic line and the overall intensity is weaker. Band $\mathrm{E}$ appears as a shoulder on the high energy side of the $4 p$ line at $23.67 \mathrm{eV}$ Figure 5 and shows no sign of the distinct maximum that is observed for ${ }^{4} \mathrm{He}$ droplets in Figures 3 and 4.

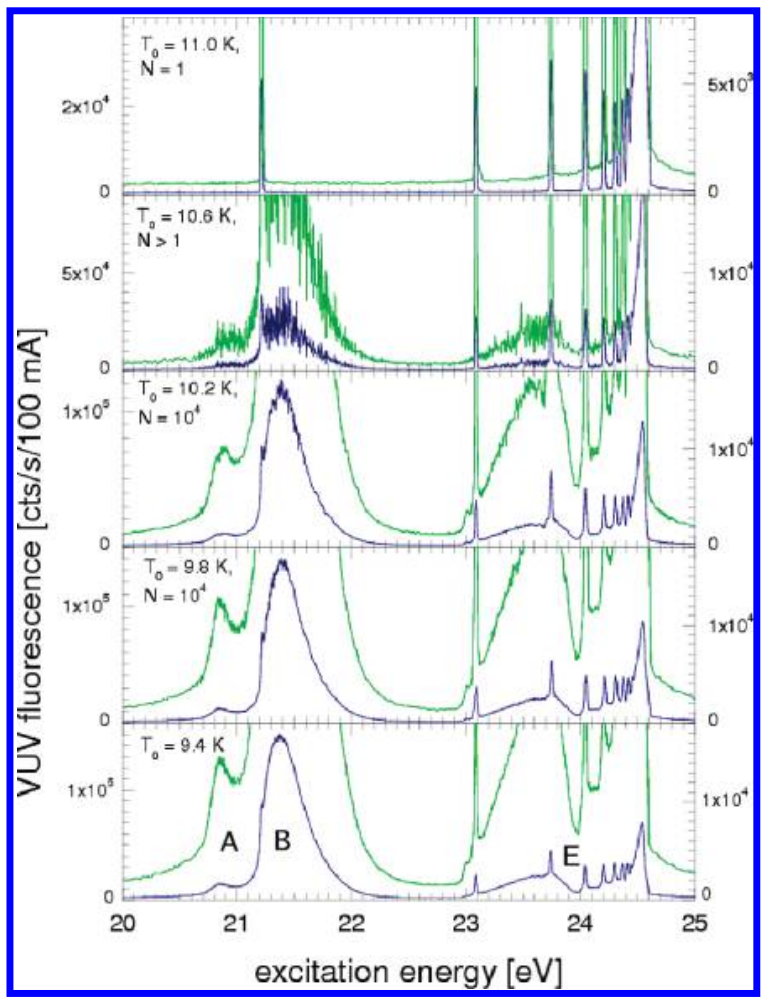

Figure 5. Fluorescence excitation spectra of ${ }^{3} \mathrm{He}$ droplets. Cluster sizes for ${ }^{3} \mathrm{He}$ were determined using a cross-jet and pick-up method. ${ }^{11} \mathrm{~A}$ rapid onset of clustering between 10.2 and $10.6 \mathrm{~K}$, consistent with ref 11 , can be seen with an average droplet size of $10^{3}$. Reference 11 reports decreasing droplet sizes with decreasing temperature. The features here show no indication of such a trend. Bands $A, B$, and $E$ are labeled. Band $E$ appears between the $4 p(23.7 \mathrm{eV})$ and $5 p(24.0 \mathrm{eV})$ line as a shoulder and has no distinct maximum unlike for ${ }^{4} \mathrm{He}$ droplets.

Short-Lived Fluorescence. Figures 7, 8, and 9 show the excitation of short-lived fluorescence of ${ }^{4} \mathrm{He}$ clusters and large droplets. Comparison with the total-fluorescence-yield excitation spectra shows a number of differences relevant for our interpretation. Band B, which evolves from a blue-shifted wing of the $2 p$ line already appears for clusters containing only $N=360$ atoms with a distinct maximum (Figure 7). It is straightforward to assign band $\mathrm{B}$ to excitations in the volume region of helium droplets where the density and the perturbation of atomic $2 p$ levels is greater than at the surface. Hence, the appearance of band $B$ at sizes smaller than seen in the total fluorescence yield spectra in Figure 1 shows that the short-lived-fluorescence excitation spectra are sensitive to excitations in the bulk region of droplets. This finding is consistent with a density-dependence of electronic decay and the following model: it is known that electronically excited helium clusters desorb electronically excited helium atoms and excimers in Rydberg states. ${ }^{5}$ This desorption is detected through efficient fluorescence in the visible, ultraviolet, and near-infrared spectral ranges that is caused by transitions between excited states of the atoms and excimers. ${ }^{5}$ The visible, ultraviolet, and near-infrared fluorescence is typically as intense as the VUV fluorescence emerging from transitions to the ground state. Normally one would expect a much stronger VUV fluorescence because the Einstein coefficient of emission scales with the third power of the transition energy. The reason for the high visible, ultraviolet, and near-infrared fluorescence efficiency is that the excitation energy is transferred to "free" 


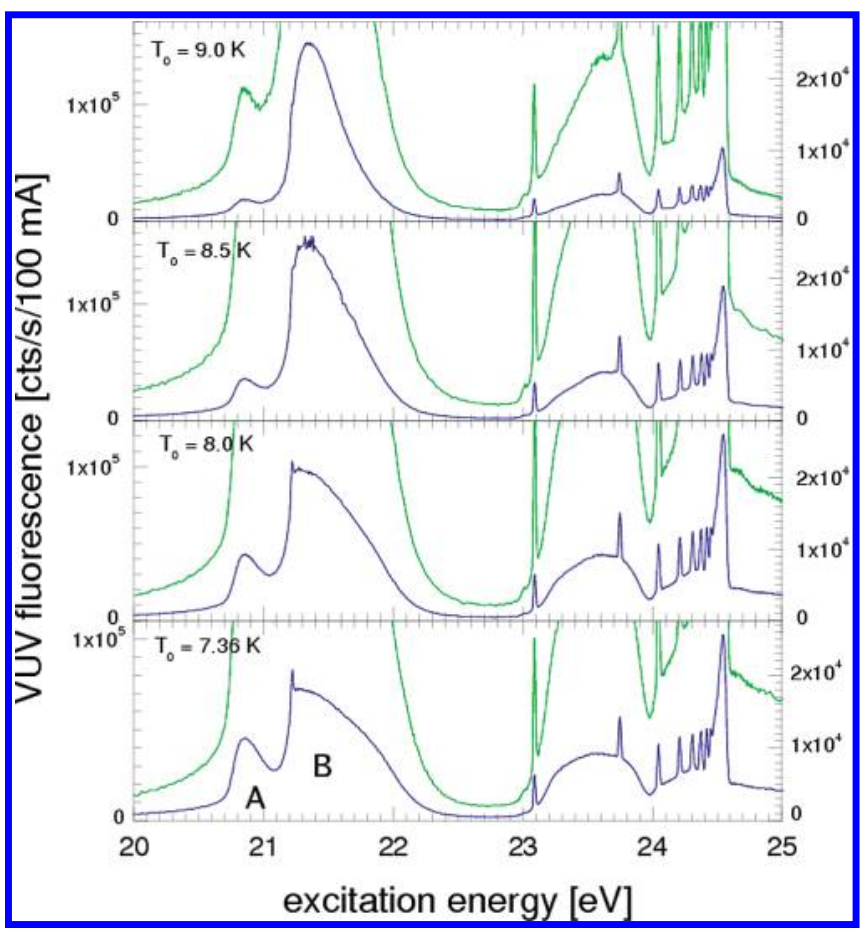

Figure 6. Fluorescence excitation spectra of ${ }^{3} \mathrm{He}$ droplets. Band A $(20.89 \mathrm{eV})$ is more separated from band B $(21.4 \mathrm{eV})$ than for ${ }^{4} \mathrm{He}$ droplet, which we interpret as being due to the lower density of liquid ${ }^{3} \mathrm{He}$ and the absence of contributions from small clusters. At stagnation temperatures lower than $8 \mathrm{~K}$ the feature at 21.4 (band B) begins to distort, indicating the formation of very large droplets.

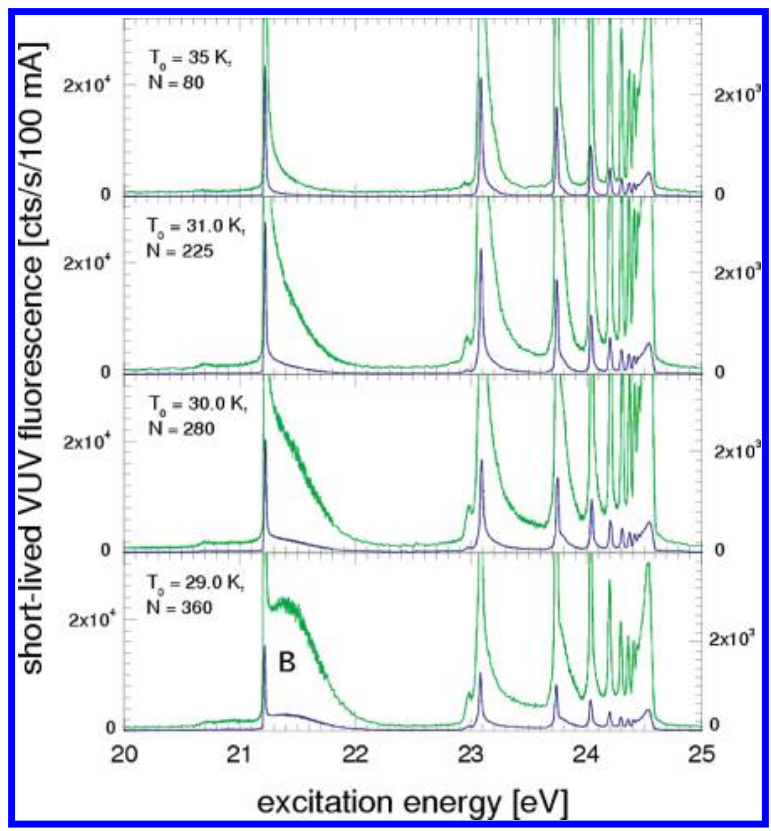

Figure 7. Excitation spectra of the short-lived fluorescence of ${ }^{4} \mathrm{He}$ droplets. A maximum of band B $(\sim 21.39 \mathrm{eV})$ can is observed for $N=$ 360 , indicating that the short-lived fluorescence excitation spectra is sensitive to excitations in the volume.

atomic and excimer states from which a direct decay to the ground state is dipole forbidden. This energy transfer is obviously much faster than the radiative lifetime of the VUV transitions

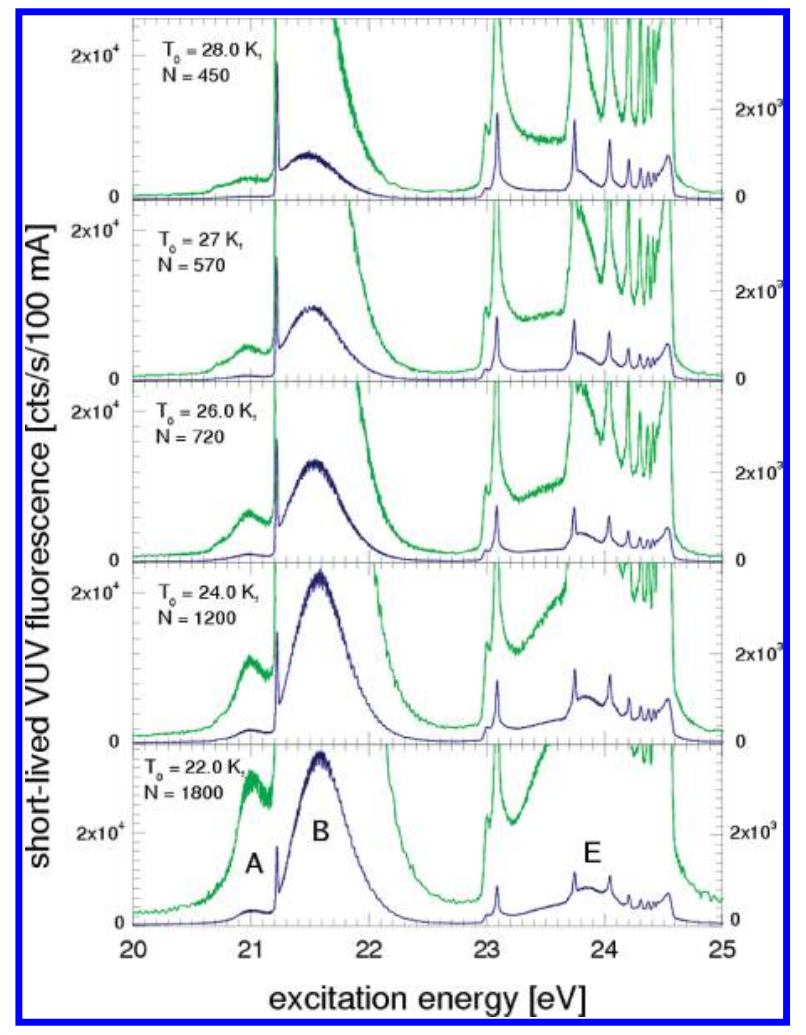

Figure 8. Excitation spectra of the short-lived fluorescence of ${ }^{4} \mathrm{He}$ droplets. Between the $2 p$ atomic line $(21.22 \mathrm{eV})$ and band B $(21.57 \mathrm{eV})$ one observes a gap that is filled in the long-lived fluorescence excitation spectra in Figure 12. The width of band B of $N=1800$ clusters is $0.49 \mathrm{eV}$.

( $\sim 1 \mathrm{~ns}$ ), indicating that the desorption efficiency is very high. Furthermore, the lines seen in the visible, ultraviolet, and nearinfrared fluorescence are unshifted with respect to free gas phase atomic and excimer emissions. ${ }^{5}$ The high absence of any shift shows that the fluorescence occurs when the atoms and excimers have already reached a great distance from the cluster/droplet surface, which provides further evidence that the desorption must be much faster than the radiative lifetime. ${ }^{5,8}$ Before fluorescence is emitted, the electronic energy tends to relax quickly (on a time scale faster than the fluorescence lifetime) to the lowest unoccupied molecular orbital (LUMO). If an excitation was located at the cluster surface desorption of excited species is more likely to happen. Consequently, in highly electronically excited helium droplets fast nonradiative relaxation competes with the desorption of highly excited Rydberg atoms and excimers and this competition depends on the location of the excitation. It is therefore plausible that excitations at the surface do not relax as much as they would if they were in the bulkvolume and produce more likely fluorescence from desorbed atoms and excimers in high-lying Rydberg states. Hence, the fluorescence lifetime is shorter for fully relaxed excitations in the volume because on average the lifetime scales with the principal quantum number of the Rydberg states as $n^{3}$.

Long-Lived Fluorescence. The above interpretation is further corroborated by the intensities found for the long-lived fluorescence excitation shown in Figures 10, 11, and 12. For $N=$ 360 , band $\mathrm{B}$ is not fully developed and shows no distinct maximum; instead, the spectrum shows a wing more characteristic for regions of lower and strongly varying density characteristic for the surface region. The shape of band B is similar to that 


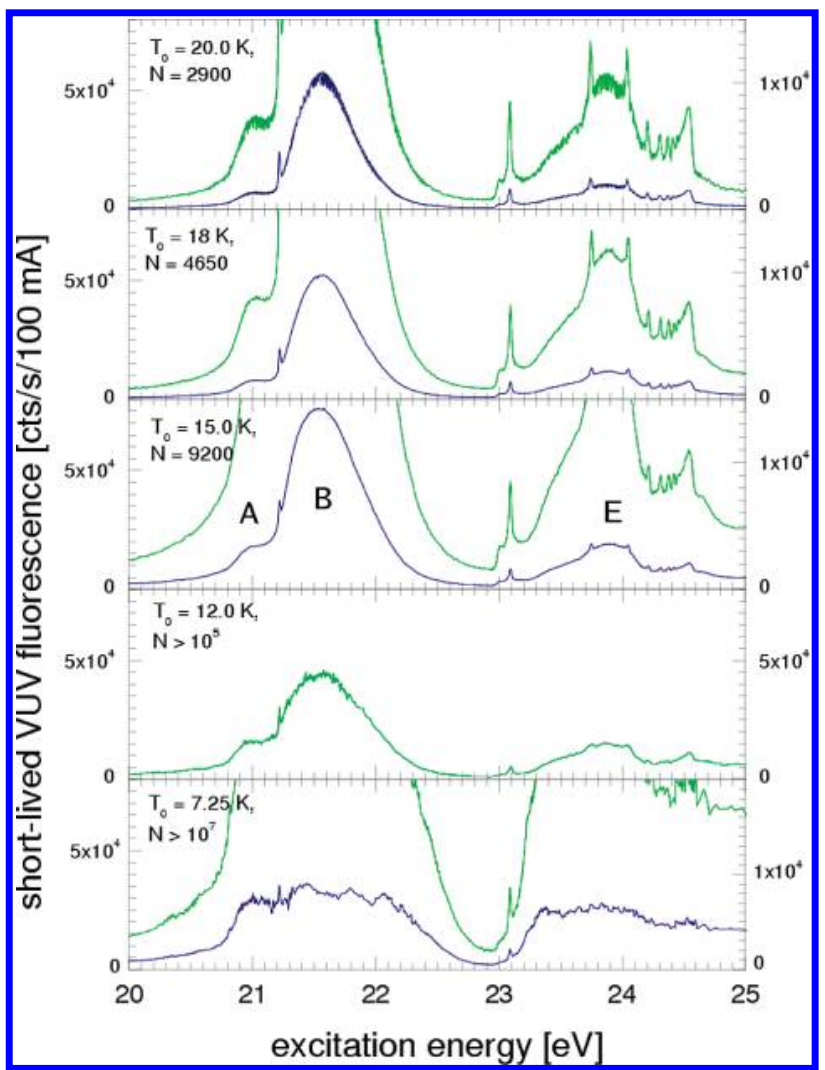

Figure 9. Excitation spectra of the short-lived fluorescence of large ${ }^{4} \mathrm{He}$ droplets.

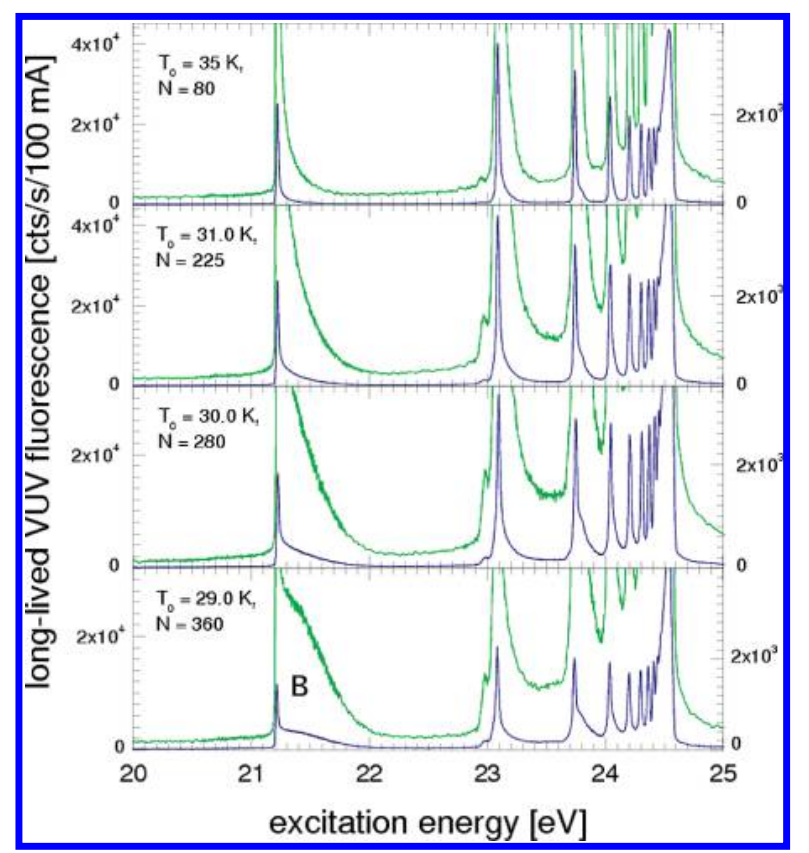

Figure 10. Excitation spectra of the long-lived fluorescence of ${ }^{4} \mathrm{He}$ droplets. The long-lived fluorescence is sensitive to regions of reduced density, that is, the surface of large droplets or small helium clusters (see text).

of the total fluorescence-yield spectrum of smaller clusters with $N=280$. Obviously, the long-lived fluorescence excitation

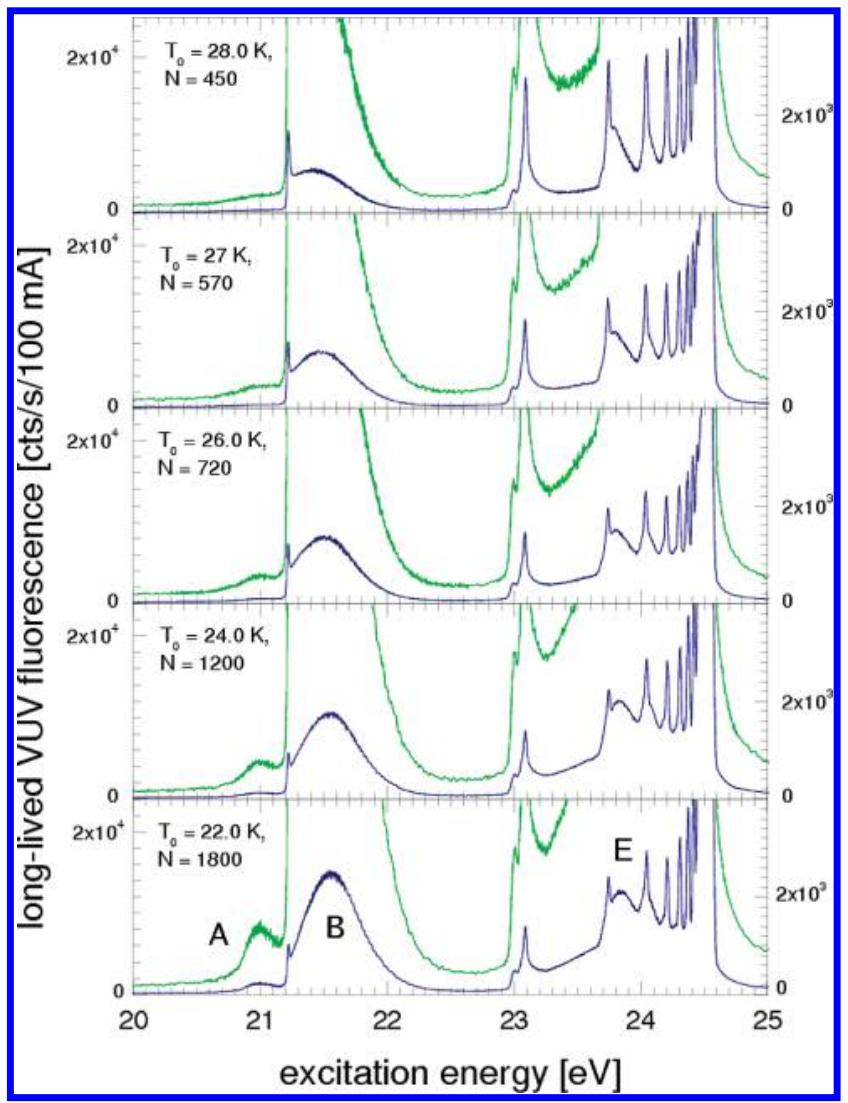

Figure 11. Excitation spectra of the long-lived fluorescence of ${ }^{4} \mathrm{He}$ droplets. Compared with the short-lived fluorescence excitation spectra bands $A$ and $B$ are less distinct. The width of band $B$ of $N=1800$ clusters is $0.53 \mathrm{eV}$, which is significantly larger than what is seen in the short-lived fluorescence spectra (Figure 8). The gap between $2 p$ atomic line and band $\mathrm{B}$ is filled, indicating contributions from low-density regions, that is, small clusters or atoms in the surface region. The opposite is observed for the maximum of band $\mathrm{E}$ which appears more distinct in the long-lived spectra (see text).

spectra are sensitive to surface excitations and show, as we would expect, trends opposite to what we observe for the short-lived fluorescence.

Features at Higher Energy $(n>3)$. In the following we discuss the features at higher energy. Here, the assignment is less clear than for the $2 s$ - and $2 p$-related bands $\mathrm{A}$ and $\mathrm{B}$, with the exception of the sharp lines, which are clearly related to helium atoms and are due to free or nearly free atoms in the molecular beam, small clusters, and loosely bound atoms at the surface, respectively. Separate bands are difficult to identify, as theoretical predictions of the nature of the high-lying bands do not exist. However, if we compare the long-lived fluorescence spectra in Figures 10-12 with the short-lived fluorescence spectra in Figures 7-9, we notice that the intensities of the features at higher energies close to the atomic ionization threshold show a clear dependence on the fluorescence lifetime. As the long-lived fluorescence spectra are more intense, we anticipate that the high-lying features are related to surface excitations. With increasing droplet size, the number of atoms in the volume increases faster than the number of atoms on the surface and one would expect that the intensities related to surface excitation decreases with respect to volume excitation. To further investigate the anticipated surface character of the high-energy excitations, we 


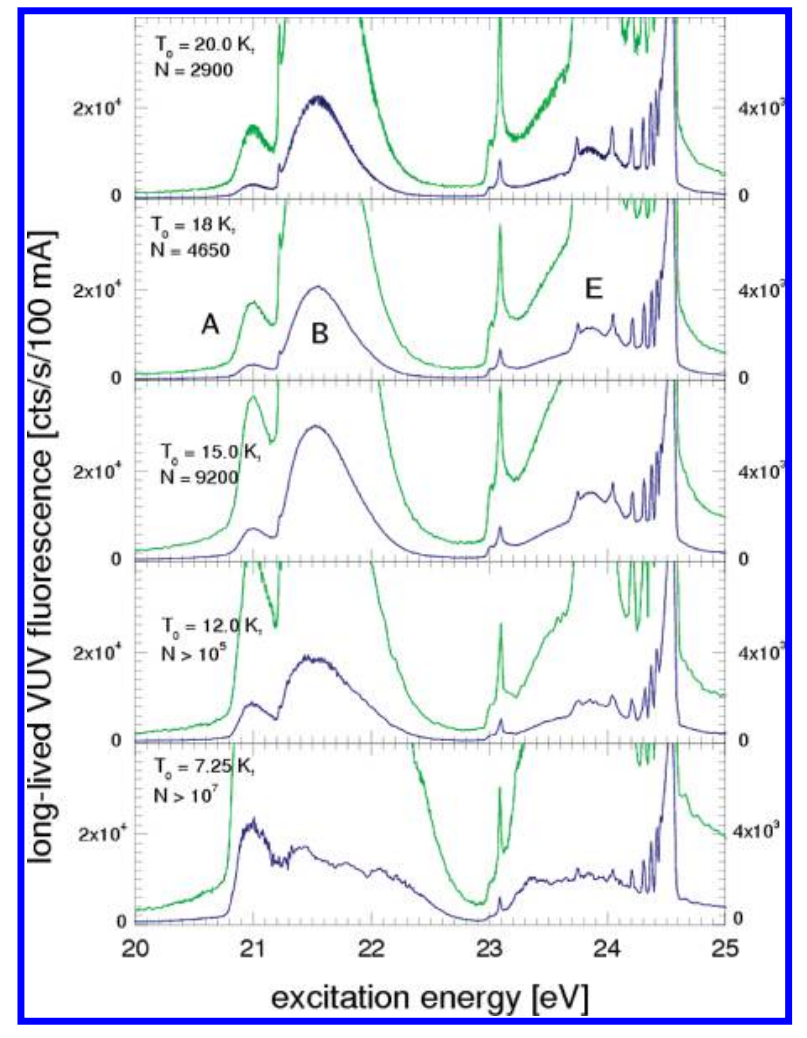

Figure 12. Excitation spectra of the long-lived fluorescence of ${ }^{4} \mathrm{He}$ droplets.

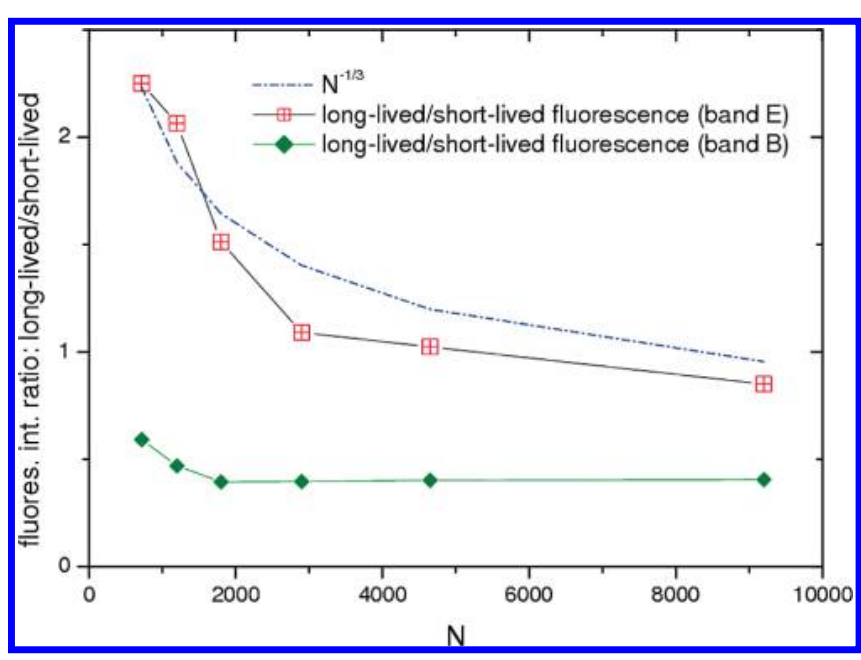

Figure 13. Ratio between the long-lived and short-lived fluorescence intensity of band $\mathrm{E}$. The same ratio is shown for band $\mathrm{B}$ for comparison. The straight dash-dotted line shows a $N^{-1 / 3}$ dependence as would be expected for an excitation located at the surface. Probing excitations located in the volume would show a constant line. The ratio between the long-lived and short-lived fluorescence is much higher for band $\mathrm{E}$ than for band $\mathrm{B}$, indicating the surface location of the excitations.

look in the following at the droplet size-dependence of the intensities. We focus on band $\mathrm{E}$ because this is the only feature at high energies for which a distinct maximum can be identified. However, the distinct maximum is only observed in the spectra of ${ }^{4} \mathrm{He}$ droplets (Figures 3, 4, 8, 9, 11, and 12), but absent for ${ }^{3} \mathrm{He}$ droplets (Figures 5 and 6). The evaluation of intensities is a difficult

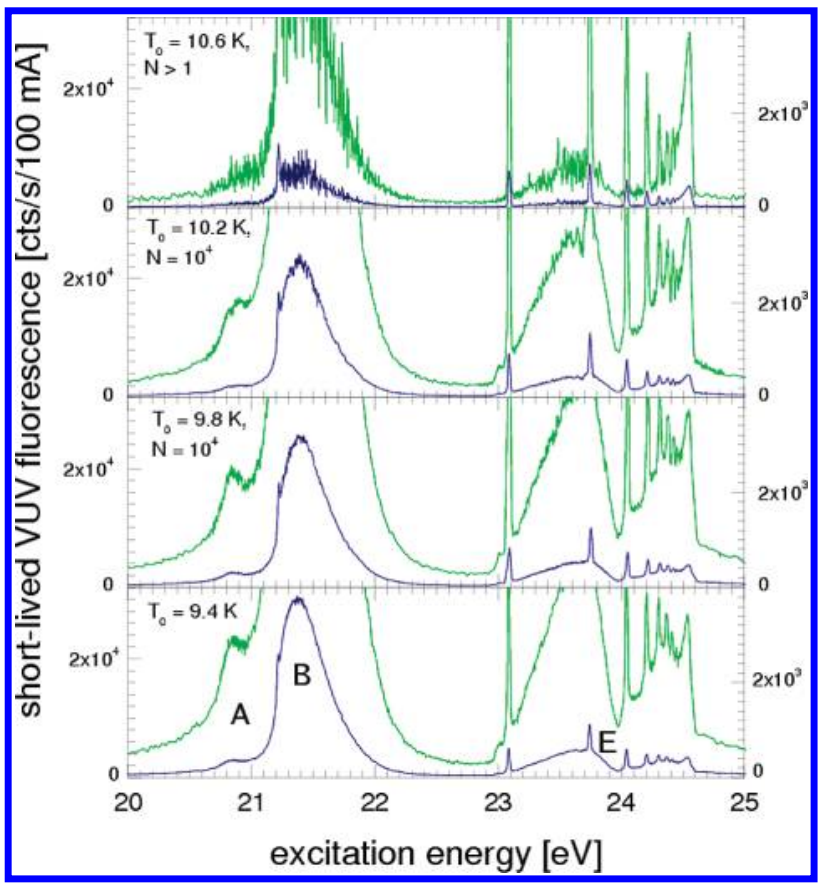

Figure 14. Excitation spectra of the short-lived fluorescence of ${ }^{3} \mathrm{He}$ droplets.

task and requires exceptional control of all experimental parameters. In our experiment, the droplet size is changed by changing the temperature of the source. Lowering the source temperature is accompanied by a flux increase; therefore, comparing intensities of spectra of different droplet sizes requires normalization, which is a procedure highly sensitive to errors. We have therefore investigated the ratio between the long-lived and the short-lived fluorescence of the maximum intensity of band E. Considering the ratio between long-lived and short-lived fluorescence eliminates the difficulties associated with normalization to the overall intensity because its contribution is canceled out. The result is shown in Figure 13. If the ratio between long-lived and short-lived fluorescence were due to volume excitation one would expect a constant line. For band $\mathrm{E}$ the intensity ratio shows a remarkably good fit to a $N^{-1 / 3}$ dependence giving strong evidence of an excitation that is located in the surface region of helium droplets. For comparison we have shown the longlived and short-lived fluorescence ratio for band $\mathrm{B}$. This ratio is much smaller than for band E, which does not surprise given that there is ample evidence for a bulk-volume nature of band B. Also, the ratio does not fit as well to $N^{-1 / 3}$ dependence as for band $\mathrm{E}$.

As all other spectral features above $23 \mathrm{eV}$ show higher intensities in the long-lived fluorescence spectra we anticipate that they are likewise due to excitations at the surface. In particular, we anticipate perturbed atomic states, of which some have previously been identified as $3 s, 3 d$, and $3 p$. $^{6}$ We see now evidence that these states reside within the surface layer. We tentatively assign band $\mathrm{E}$ to an $n=4$ related excitation, but further evidence is required. The lack of intensity of high-lying excitations located in the bulk volume is consistent with the spectral features recorded using a solar-blind photomultiplier, as reported earlier. ${ }^{8}$ The solar-blind multiplier is sensitive to fluorescence originating from the bulk-volume region and the spectrum reported shows many similarities with the short-lived fluorescence excitation spectrum, the features above $23 \mathrm{eV}$ being even weaker. We interpret this result such that in bulk liquid helium 


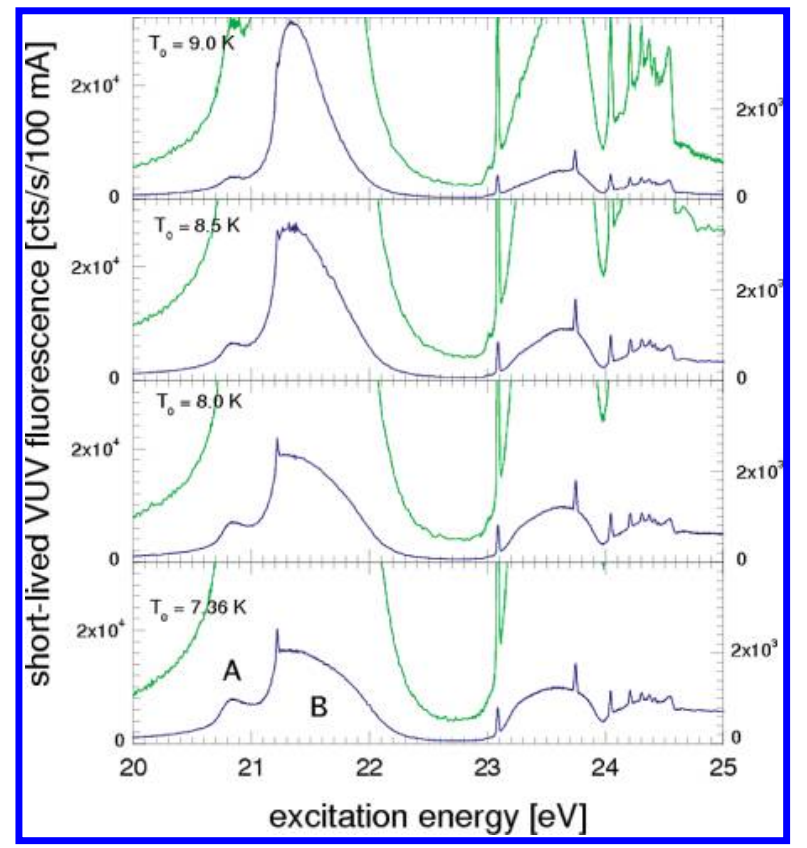

Figure 15. Excitation spectra of the short-lived fluorescence of ${ }^{3} \mathrm{He}$ droplets.

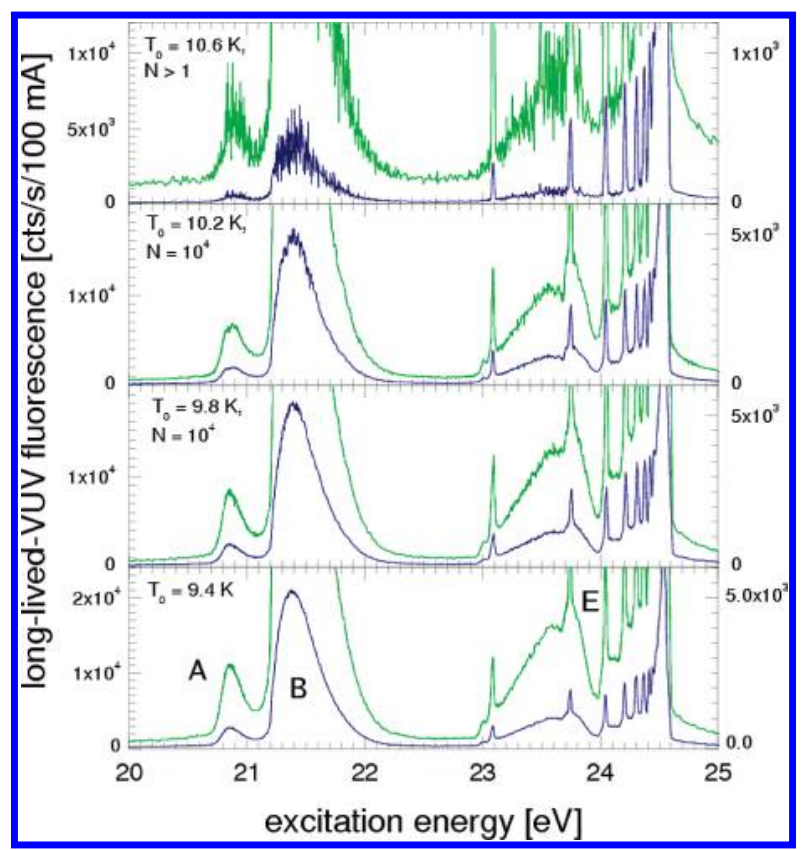

Figure 16. Excitation spectra of the long-lived fluorescence of ${ }^{3} \mathrm{He}$ droplets. We observe higher intensities of the bands at energies higher than $23 \mathrm{eV}$ compared to the intensity of band B than for short-lived fluorescence excitation spectra. This shows that the "high-energy" bands correspond to regions of lower density, that is, atoms in the surface layer. Contributions of small clusters can be excluded for ${ }^{3} \mathrm{He}$ droplet beams (see text).

Rydberg states higher than $n=2$ are destabilized due to the Pauli repulsion and the low polarizability of bulk liquid helium. A set of orthogonal states extending beyond the average internuclear separation are therefore difficult, if not impossible to construct in an infinite, disordered system like bulk liquid helium, but likely to exist at the surface of liquid helium and helium droplets. For

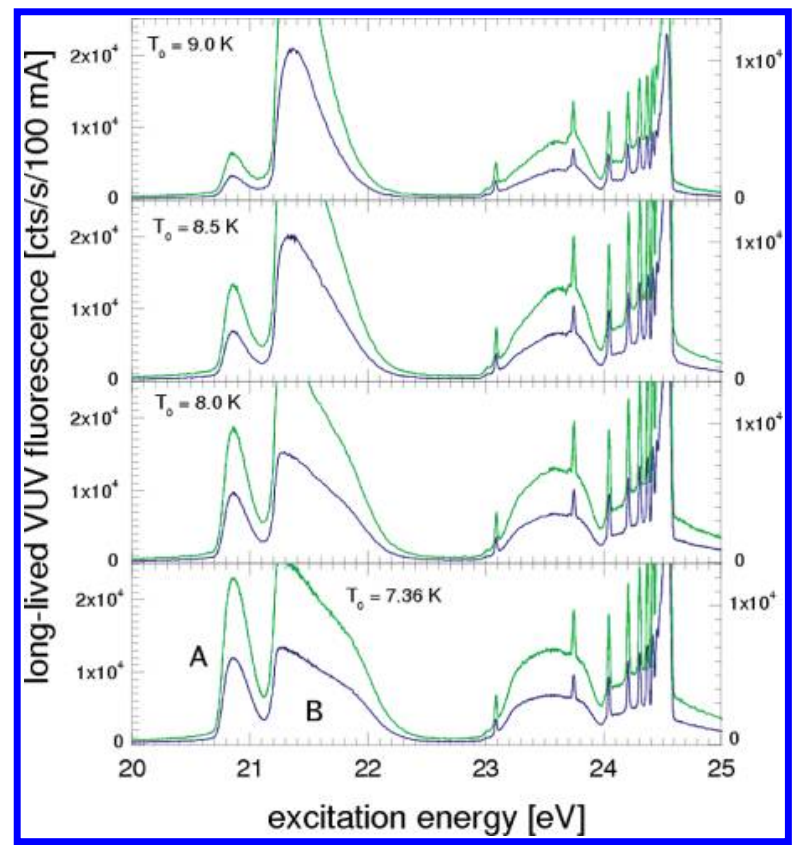

Figure 17. Excitation spectra of the long-lived fluorescence of ${ }^{3} \mathrm{He}$ droplets. Band A $(20.89 \mathrm{eV})$ is more separate from band B than observed for the short-lived fluorescence excitation spectra of ${ }^{3} \mathrm{He}$ droplets. This shows that it is possible to selectively probe regions of reduced density by measuring the long-lived fluorescence yield.

small helium clusters high Rydberg states may exist because they expand beyond the entire cluster.

It is interesting to compare this finding with the long-lived/ short-lived intensity ratio found for ${ }^{3} \mathrm{He}$ droplets (see Figures 14-17). As spectra of small ${ }^{3} \mathrm{He}$ clusters are not available the size dependence cannot be investigated. However, ${ }^{3} \mathrm{He}$ droplets provide a convenient benchmark for density effects. We observe higher intensities of the bands at energies higher than 23 $\mathrm{eV}$ compared to the intensity of band $\mathrm{B}$ than for the total fluorescence (Figure 3) and the short-lived fluorescence excitation spectra (Figure 9). The spectral region of band $\mathrm{E}$ shows no distinct maximum as for ${ }^{4} \mathrm{He}$ droplets other than a wing decreasing in intensity. We interpret this wing as due to the perturbation in the surface region being even lower for ${ }^{3} \mathrm{He}$ droplets than for ${ }^{4} \mathrm{He}$ droplets. At $23.78 \mathrm{eV}$, just above the $4 p$ line the long-lived/ short-lived fluorescence ratio, being indicative for features located in low-density regions, is two, which is similar to that of the small and medium sized ${ }^{4} \mathrm{He}$ clusters in Figure 13.

The fact that the maximum of band $\mathrm{E}$ appears for ${ }^{4} \mathrm{He}$ clusters larger than $N=450$ and that ${ }^{3} \mathrm{He}$ droplets only exhibit a blueshifted wing shows that a minimum density is required to produce band $\mathrm{E}$. We therefore conclude that the excitation is buried within the surface layer. This is supported by the observed energy shift shown in Table 1 . Different to band B, where the shift saturates at $N=1200$, the shift continues up to $N=9200$. Such a behavior is expected for an extended electronic wave function with $n \geq 3$, which is perturbed by dense helium. If the excitation was located in the outer surface region both excited state electron density and ground state electron density of the perturbing atoms do not have sufficient overlap to produce notable perturbation. ${ }^{9}$

In conclusion, we have presented fluorescence excitation spectra of ${ }^{3} \mathrm{He}$ and ${ }^{4} \mathrm{He}$ clusters and droplets over a large size 
range. The fluorescence excitation was recorded in "time-windows" specific to short-lived and long-lived fluorescence allowing us to better assign the spectral features. We analyze spectral shifts with respect to atomic resonance lines and identify excitations located in regions of weak and strong perturbation, that is, surface states and bulk-volume states, respectively. We show that shortlived fluorescence is sensitive to regions of large perturbation, that is, wave functions with high electron density in regions of increased density, that is, excitations in the bulk region of large droplets, whereas long-lived fluorescence is sensitive to small clusters or the surface region. The observed size dependence of short-lived and long-lived fluorescence shows unambiguously a location of high-lying features within the surface region. This points toward quenching of high-lying electronic states in liquid helium, a possible reason being that the perturbation due to Pauli repulsion is too strong and the polarizability too low to build up orthogonal wave functions. Hence, most of the oscillator strength in bulk liquid helium is concentrated in the $2 s$ and $2 p$ excitations, while at the surface of helium droplets and in small helium clusters high-lying Rydberg states exist.

\section{AUTHOR INFORMATION}

\section{Corresponding Author}

*E-mail: kvh6@le.ac.uk.

\section{ACKNOWLEDGMENT}

We are grateful to Mark Watkins for critically reading the manuscript.

\section{REFERENCES}

(1) Wörmer, J.; Joppien, M.; Zimmerer, G.; Möller, T. Phvs. Rev. Lett. 1991, 67, 2053-2056.

(2) Wörmer, J.; Karnbach, R.; Joppien, M.; Möller, T. L. Chem. Phvs. 1996, 104, 8269-8278.

(3) Joppien, M.; Karnbach, R.; Möller, T. Phvs. Rev. Lett. 1993, 71, 2654-2657.

(4) Joppien, M.; Müller, R.; Möller, T. Z. Phvs. D: At. Mol. Clusters 1993, 26, 175-177.

(5) von Haeften, K.; de Castro, A. R. B.; Joppien, M.; Moussavizadeh L.; von Pietrowski, R.; Möller, T. Phvs. Rev. Lett. 1997, 78, 4371.

(6) von Haeften, K.; Laarmann, T.; Wabnitz, H.; Möller, T. Phys. Rev. Lett. 2001, 87, 153403.

(7) von Haeften, K.; Laarmann, T.; Wabnitz, H.; Möller, T. $\underline{P h y s . R e v .}$ Lett. 2002, 88, 233401.

(8) von Haeften, K.; Laarmann, T.; Wabnitz, H.; Möller, T. $\underline{\text { LPhys. B: }}$ At. Mol. Opt. Phvs. 2005, 38, S373-S386.

(9) von Haeften, K.; Fink, K. Eur. Phys. I. D 2007, 43, 121-124.

(10) Closser, K.; Head-Gordon, M. J. Phys. Chem. A 2010, 2622-2648.

(11) Harms, J.; Toennies, J.; Barranco, M.; Pi, M. Phvs. Rev. B 2001, 63, 184513.

(12) Penanen, K.; Fukuto, M.; Heilmann, R.; Silvera, I.; Pershan, P. Phvs. Rev. B 2000, 62, 9621-9640.

(13) Harms, J.; Toennies, J. P.; Dalfovo, F. Phvs. Rev. B 1998, $58,3341$.

(14) Karnbach, R.; Joppien, M.; Stapelfeldt, J.; Wörmer, J.; Möller, T. Rev. Sci. Instrum. 1993, 64, 2838-2849.

(15) von Pietrowski, R.; Rutzen, M.; von Haeften, K.; Kakar, S.; Möller, T. Z. Phvs. D: At. Mol. Clusters 1997, 40, 22-24.

(16) von Haeften, K.; Laarmann, T.; Wabnitz, H.; Moller, T. I. Electron Spectrosc. Relat. Phenom. 2000, 106, 199-206.

(17) Fröchtenicht, R.; Henne, U.; Toennies, J. P.; Ding, A.; FieberErdmann, M.; Drewello, T. I. Chem. Phvs. 1996, 104, 2548.
(18) Peterka, D. S.; Lindinger, A.; Poisson, L.; Ahmed, M.; Neumark, D. M. Phvs. Rev. Lett. 2003, 91, 043401.

(19) Peterka, D.; Kim, J.; Wang, C.; Neumark, D. I. Phvs. Chem. B 2006, 110, 19945-19955.

(20) Peterka, D.; Kim, J.; Wang, C.; Poisson, L.; Neumark, D. L. Phvs. Chem. A 2007, 111, 7449-7459.

(21) Kornilov, O.; Wang, C.; Bünermann, O.; Healy, A.; Leonard, M.; Peng, C.; Leone, S.; Neumark, D.; Gessner, O. I.Phys. Chem. A 2009, 114, 1437-1445.

(22) Schwentner, N.; Koch, E.; Jortner, J. Electronic Excitations in Condensed Rare Gases; Springer: New York, Berlin, 1985.

(23) Wasilewski, J. Int. I. Quantum Chem. 1989, 36, 503.

(24) Meier, U.; Staemmler, V. Theor. Chim. Acta 1989, 76, 95.

(25) Fink, R.; Staemmler, V. Theor. Chim. Acta 1993, 87, 129.

(26) Jungen, M. I. Chem. Phvs. 1981, 74, 750-751.

(27) Jungen, M. Theor. Chim. Acta 1981, 60, 369.

(28) Huzinaga, S. Approximate Atomic Functions. I; University Alberta, Canada.

(29) Kaufmann, K.; Baumeister, W.; Jungen, M. I. Phvs. B 1989, 22, 2223.

(30) Hagena, O. F. Cluster Ion Sources 1992, 2374-2379.

(31) Guzielski, V.; Castex, M.; Wörmer, J.; Möller, T. Chem. Phys. Lett. 1991, 179, 243-246.

(32) von Haeften, K.; Metzelthin, A.; Rudolph, S.; Staemmler, V.; Havenith, M. Phvs. Rev. Lett. 2005, 95, 215301.

(33) Harms, J.; Toennies, J.; Knuth, E. I. Chem. Phvs. 1997, 106, 3348.

(34) Buchenau, H.; Knuth, E.; Northby, J.; Toennies, J.; Winkler, C. I. Chem. Phvs. 1990, 92, 6875.

(35) Henne, U.; Toennies, J. I. Chem. Phys. 1998, 108, 9327. 